\title{
Review of: "Evaluation of the Biological Effects of Novel Tobacco Product Vapor Using Two- or Three- Dimensional Culture Systems of Human Bronchial Epithelia"
}

\author{
Naheem Yaqub $^{1}$, Wenhui Song ${ }^{1}$ \\ 1 University College London, University of London
}

Potential competing interests: The author(s) declared that no potential competing interests exist.

Mori et al (2021) tested the effects of aqueous exposure (AqE) of a novel tobacco vapour product (NTV) created by a new method of tobacco inhalation in this comparative study. The study was funded and conducted by the tobacco company currently involved in commercialising a line of heated tobacco products. The authors conclude that NTV vapour has fewer oxidative stress effects with limited effect on tissue inflammation in the 2D and 3D models of the respiratory epithelium, respectively, when compared to aqueous extract of 3R4F reference cigarette smoke.

The study chose to compare the AqE concentration of NTV and 3R4F products based on nicotine concentration instead of other measures such as total particulate matter. The NTV used for 2D studies contained a higher concentration of nicotine compared to 3R4F (x100 times higher nicotine content). It was concluded that the NTV vapour had less of an effect on oxidative stress compared to the 3R4F reference at this concentration in 2D. This conclusion was based on the data presented which showed reduced toxicity after 24 hours exposure to NTV vs 3R4F in cell viability ( $\sim 60 \%$ viability at the maximum concentration for NTV compared to $\sim 20 \%$ for 3R4F), and no significant change in oxidative stress measured using a GSH/GSSG assay (compared to $~ 100 \%$ reduction in GSH at maximum exposure concentration) after 2 hours of exposure. However, the NTV induced a dose-dependent increase in reporter gene activity (analogous to increased oxidative stress) in the ARE reporter assay. But as the concentration of NTV (puffs $/ \mathrm{ml}$ ) required to elicit this response was $\sim 1000 x$ higher when compared to the reference vapour, the study concludes that the NTV had a comparatively reduced effect on cellular oxidative stress.

The effect of NTV on metabolic processes remains unclear. Dose-dependent effects on metabolic processes can be direct or via changes in proliferation rates or cell cycle. This may increase or decrease the metabolism of the NTV or 3R4F tobacco toxins, which may affect the cytotoxicity outcomes. Therefore, the study of the metabolic effects of NTV should provide additional insight into the effect on cell viability. The cytotoxicity to both compounds shown in the 2D model was not replicated in the 3D respiratory epithelium model. The difference in results between 2D and 3D model systems is not unexpected given the profound experimental and biological differences between these models. As cytotoxicity assays are primarily effective to determine a compound's ability to kill or severely damage cells, the effects produced by sub- 
lethal doses of NTV cannot be assumed to be proportional in any way to a dose producing explicit cytotoxicity ${ }^{1}$. Also, the carcinogenicity of NTV is yet to be investigated as its mechanism and correlation with the cytotoxic effect remains elusive, especially in the sub-lethal dose test conditions presented. For 3D studies, a relatively equal concentration of nicotine was used (a new serial dilution method was employed for infusing media with NTV instead of direct infusion due to protocol limitations). At concentrations determined to be non-cytotoxic via histology and adenylate kinase toxicity assays after 24 hours of exposure, cytokine measurements and microarray analysis showed key changes in the expression profiles in the 3D airway model after 3R4F and NTV exposure. The study showed that after 24 hours of exposure, 2 puffs/ml NTV produced significantly fewer differentially expressed genes (25 DEGs) when compared to $0.2 \mathrm{puffs} / \mathrm{ml}$ of the 3R4F reference vapour (434 DEGs). Similar results were observed with regards to the expression of inflammatory mediators, of NTV exposure to 3D cultures, where after 24 hours, IFN-y was significantly upregulated, in comparison to TNF-a, IFN-C, and IL-1b, IL-8, VEGF-A, IL-7 and TARC upregulation for 3R4F reference vapour. Interestingly, VEGF decreased with NTV exposure, and cytokine expression levels did not increase linearly with increasing concentrations of 3R4F reference exposure, which was not discussed by the authors.

The study characterised the toxicity of the NTV in 2D and 3D models of the respiratory epithelium. There are inherent limitations to the use of the virally transformed BEAS-2B cells for investigating the respiratory epithelium. These cells maintain their ability to undergo squamous differentiation in vitro in response to foetal bovine serum or tumour growth factor-beta ${ }^{2}$. Though they do form primary cilia, they do not form motile cilia which are characteristic of the native airways. They also fail to sufficiently express tight junctions and subsequently have low TEER similar to A549 cells, and lack expression of mucins and numerous CYP enzymes ${ }^{3}$. Perhaps more worryingly, recently researchers showed BEAS-2B cells share characteristic cell surface markers as well as other features with mesenchymal stem cells, therefore questioning the epithelial origins of the immortalised BEAS-2B cells ${ }^{4}$. Despite this, BEAS-2B cells have been used to analyse the effect of cigarette smoke ${ }^{5}$ and particulates in the airways as well as mechanistic and toxicological studies ${ }^{6}$ and cytotoxicity studies of electronic cigarette fluids ${ }^{7}$. The study also used a commercial 3D model of the respiratory epithelium which serves as a relevant translational model of the respiratory epithelium and this choice should be commended.

The use and applicability of the 2D model were chosen due to its ability to characterise oxidative stress responses in previous interlaboratory experts. Another approach for the characterisation of toxicity of novel compounds would be to select assays based on their ability to detect previously unknown toxicities. The 3D model which was used has far more benefits to characterising toxicity in the function, morphology and biological changes to the respiratory epithelium which were not utilised in this study e.g., epithelial barrier measurements such as TEER or molecule permeability assays, immunofluorescent imaging and quantification of relevant protein markers such as ZO-1, E-cadherin, acetylated alpha-tubulin etc., motile cilia analysis via recording and quantification of ciliary beta frequency. These assays may have provided additional information to the cytotoxicity assays conducted in 2D and 3D. The microarray analysis in 3D 
compared to the reference 3R4F was informative in comparative terms. A short exposure time of 24 hours was used which is commonplace for toxicology assays, studying immediate effects, whereas cancer and other toxicities from cigarette smoke in humans develop with prolonged exposure. The ability of current in vitro models to be able to show this effect can be debated, however, the exposure time may significantly affect the dose-dependent response of compounds in vitro. Therefore, before moving into in vivo experiments, longer-term studies at higher concentrations of NTV in 3D models of the respiratory epithelium, whilst taking advantage of the multiparametric forms of analysis offered by these models, should be carried out to characterise the metabolic and biological effects of NTV.

\section{References:}

1. Wan, J. et al. Evaluation of In Vitro Assays For Assessing the Toxicity of Cigarette Smoke and Smokeless Tobacco. Cancer Epidemiol. Biomarkers Prev. 18, 3263 (2009).

2. Huang, S., Wiszniewski, L. \& Constant, S. The Use of In Vitro 3D Cell Models in Drug Development for Respiratory Diseases. in Drug Discovery and Development - Present and Future (InTech, 2011). doi:10.5772/28132.

3. Prytherch, Z. \& Berube, K. Modelling the Human Respiratory System: Approaches for in Vitro Safety Testing and Drug Discovery. in Human-based systems for translational research (eds. Coleman, R. \& Fox, D.) 66-87 (Royal Society of Chemistry, 2014).

4. Han, X., Na, T., Wu, T. \& Yuan, B.-Z. Human lung epithelial BEAS-2B cells exhibit characteristics of mesenchymal stem cells. PLoS One 15, e0227174 (2020).

5. Dugour, A., Elías, F. \& Figueroa, J. Harmfull effects of cigarette smoke on a respiratory epithelium line (Calu 3): Prevention by N-acetylcisteine. Eur. Respir. J. 42, (2013).

6. Ong, H. X., Traini, D. \& Young, P. M. Pharmaceutical applications of the Calu-3 lung epithelia cell line. Expert Opin. Drug Deliv. 10, 1287-1302 (2013).

7. Hua, M. et al. Identification of Cytotoxic Flavor Chemicals in Top-Selling Electronic Cigarette Refill Fluids. Sci. Rep. 9, 2782 (2019). 